\title{
Design and Performance Analysis of UWB MIMO OFDM System Using Microstrip Antennas
}

\author{
Harshal Nigam*, Mithilesh Kumar* \\ *Electronics Engineering Department, University College of Engineering, Rajasthan Technical University, Kota Rajasthan \\ India
}

hrshInigam@gmail.com, mith_kr@yahoo.com

\begin{abstract}
OFDM based Ultra Wideband (UWB) system combines OFDM modulation technique with a multi banding approach, which divides the spectrum into several sub-bands, whose bandwidth is approximately $528 \mathrm{MHz}$. It is a popular method for high data rate wireless communications. MIMO techniques promise a significant boost in the performance of OFDM systems. In this paper, a novel design UWB MIMO OFDM system using a compact double-sided printed antenna having ultra-wideband and band-notched characteristic is proposed. The UWB antennas are designed on CST Microwave studio simulation software with return loss less than $-10 \mathrm{~dB}$, and further using the results from CST, OFDM transmission and reception on a MIMO system is done on MATLAB software. The MIMO UWB antennas are designed using polarization diversity of the individual antennas. The performance of this system is studied using BPSK modulation and finally bit error rate and operating range is obtained. The transmitting bandwidth is UWB (i.e. 3.1-10.6GHz) and the receiving bandwidth is notch UWB (notch bandwidth $5-5.9 \mathrm{GHz}$ ) are chosen to reduce the interference at the receiver side. Furthermore, a MIMO UWB system is designed at a frequency of $9.4 \mathrm{GHz}$ that is used for micro power radar application in the ultra wideband removing the interference from WLAN band.
\end{abstract}

Keywords-Band-notched, Bit Error Rate (BER), Doublesided Printed, Multiple Input Multiple Output (MIMO), Orthogonal Frequency Division Multiplexing (OFDM)

\section{INTRODUCTION}

In wireless communications, spectrum is a scarce resource and hence imposes a high cost on the high data rate transmission. Fortunately, the emergence of multiple antenna system has opened another very resourceful dimension space, for information transmission in the air. It has been demonstrated that multiple antenna system provides very promising gain in capacity without increasing the use of spectrum, reliability, throughput, power consumption and less sensitivity to fading, hence leading to a breakthrough in the data rate of wireless communication systems. Since then, multiple input multiple output (MIMO) system has become one of the major focuses in the research community of wireless communications and information theory as in [1]. We use ultra wideband (UWB) orthogonal frequency division multiplexing (OFDM) along with MIMO system. OFDM is a multicarrier system, the available spectrum is divided into many narrow bands and data is divided in parallel streams each transmitted on a separate band. OFDM gives a simple way of dealing with multipath as in [2]. The applications of OFDM include Digital Television, Asymmetric Digital subscriber loop (ADSL) and future mobile telephony.

The UWB antenna has its advantages in the areas of radar system, communication area and military applications. The Federal Communications Commission (FCC) has approved on the 3.1-10.6 GHz unlicensed band for UWB communication since 2002 which has overcast the band of WLAN (5.15$5.825 \mathrm{GHz}$ ) and may interfere with some communication systems as in [3]. So, if the band-notched frequency of WLAN can be produced, the interference could be avoided.

In this paper, a simple and compact planar Ultra Wideband Printed patch antenna with a U-shaped slot is designed having band notched characteristic that shows acceptable return loss and bandwidth over UWB frequency band as in [4]. It overcomes the interference of the UWB and the WLAN.

This UWB antenna is further used in a MIMO system having two transmit and two receive antennas and then a performance analysis of the system is done using orthogonal frequency division multiplexing technique over ultra wideband.

When multiple antennas are involved at closer spacing the technical challenges are more pronounced compared to a SISO (Single Input Single Output) system. Hence, the basic aim of MIMO antenna design is to minimize the correlation between the multiple signals as in [5]. The parameter that describes the correlation between the received signals is mutual coupling, as it may affect the performance of the system. By calculating the mutual coupling, one can analyse the electromagnetic field interactions that exist between antenna elements of a MIMO system. The mutual coupling mainly depends on the distance between the elements of an antenna array. The distance between antenna elements in practice cannot be extended beyond a certain level which limits the use of spatial diversity to achieve the desired spectral efficiencies and transmission qualities. As an alternative solution to achieve compactness in MIMO systems, the use of pattern diversity as in $[6,7]$, multimode diversity as in [8], and polarization diversity techniques as in [9] in conjunction with space diversity are discussed in the literature. In the present design, the orthogonal polarization concept is 
applied to the proposed multi slot patch antenna yielding better results in terms of return loss and mutual coupling.

In OFDM approach, the spectrum is divided into 14 bands (each with a bandwidth equal to $528 \mathrm{MHz}$ ), and devices are allowed to statically or dynamically select which bands to use for transmission. The multi channel modulation divides a given channel into many parallel sub-channels or sub-carriers, so that multiple symbols are sent in parallel so that each subcarrier experience a flat channel as in [10].

This paper is organized as follows. In Section II, the design methodology and structure of a single UWB antenna along with a notch is described along with the simulation results. Section III incorporates the use of above antenna in a $2 \mathrm{X} 2$ MIMO system. Polarization diversity is applied to the above MIMO antennas and simulated results are analysed further. In Section IV the above MIMO system is used for OFDM transmission and reception and the overall results are combined to study the performance analysis of the given system. Section V concludes the design of our system.

\section{UWB ANTENNA DESIGN}

\section{A. Design Approach}

The UWB antenna is designed on a substrate which is printed on both sides, on one side is the patch and other side is a partial ground plane. The patch is fed by a micro strip feed line, appropriate matching of the feed line is required to produce UWB characteristics of the antenna.

The notched bands are generated by conventional resonant structures, that is the slots or uniform half wavelength resonators, the spurious notched bands caused by spurious resonances would emerge and distort the antenna's UWB performance. When exciting the slot mode, a strong resonance is provoked at $5.5 \mathrm{GHz}$, which alters the current distribution within the planar surface of the monopole, in turn translates into the band notch. The band-notched frequency produced by the unmatched impedance is $5.5 \mathrm{GHz}$. The length of the slot is half wavelength of the notch frequency, which can be calculated as

$$
L=\frac{c}{2 f \sqrt{e}}
$$

As observed in (1), c represents the velocity of light, $\mathrm{f}$ represents the notch frequency e represents the equivalent dielectric constant. Basically, the width of the notch band lies on the width of the slot and changing the length of the slot can cause the change on notch frequency. That is, the wider and longer the slot, the wider notch band and the higher notch frequency.

\section{B. Antenna Structure}

The geometry of the given UWB antenna is illustrated in Figure 1. It is fabricated on a $64 \times 30 \mathrm{~mm}^{2} \mathrm{FR}-4$ substrate with a dielectric constant of 4.3 and a substrate thickness of 1.5 $\mathrm{mm}$. The top and bottom patches printed on the substrate are the radiating structure and the ground plane. The back view is shown in Figure 2.

The top patch of the substrate has dimension of $9 \times 10.5 \mathrm{~mm}^{2}$ which is fed by a strip line having a width of $2.9 \mathrm{~mm}$. The bottom patch of substrate is just a partial ground plane which generates ultra wideband characteristics of the antenna, band notched characteristics of the antenna are generated by cutting a U-slot on the upper patch as shown in Figure 3. The dimensions of the slot are also shown in the same. The proposed antenna with and without U-shaped slot has been simulated by CST Microwave studio.

\section{Antenna Simulation Analysis}

The simulation results of the antenna are shown in Figure 4, from the simulated graph it is observed that for UWB frequency range 3.1-10.6 GHz, $\mathrm{S}_{11}<-10 \mathrm{~dB}$. Thus the results are in agreement that antenna operates on UWB.

The notched results are shown in Figure 5, which shows that the WLAN frequency band 5.1-5.9 GHz has been notched. The comparative results of antenna with and without notch are shown in Figure 6.

From the UWB antenna simulation curve of Figure 4 it can be seen that the smallest attenuation that is $S_{11}=-23 \mathrm{~dB}$ occurs for a frequency of $9.4 \mathrm{GHz}$, so we select this frequency.

MIMO UWB system is designed at this frequency of $9.4 \mathrm{GHz}$ that is used for micro power radar application in the ultra wideband removing the interference from WLAN band.

\section{III.MIMO SYSTEM DESIGN}

\section{A. Design of Two Element MIMO Transmitting Section with Polarization Diversity}

Consider, a 2X2 MIMO system having two antennas at the transmitter side and two at the receiver side. The main criteria for MIMO system design is mutual coupling, which mainly arises due to the smaller spacing between the two antennas, when multiple antennas are involved at closer spacing the design issues are more complicated. The mutual coupling mainly depends on the distance between the elements of an antenna array.

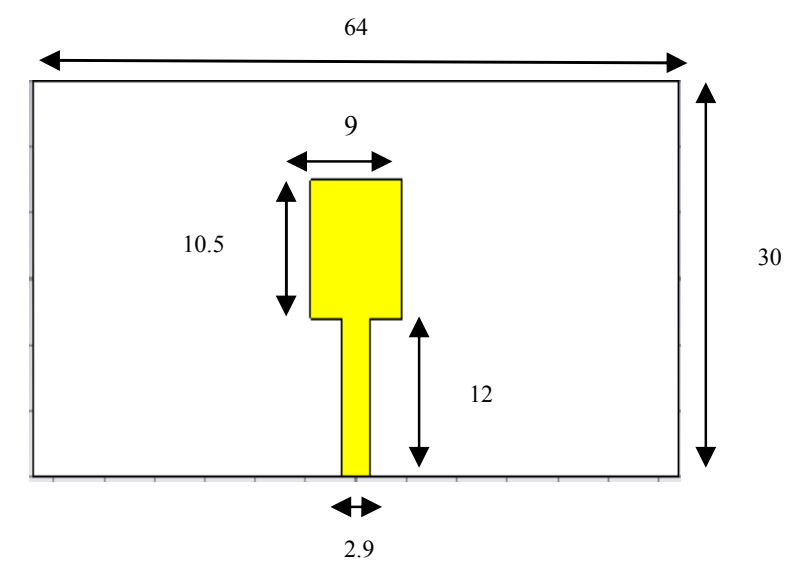

Figure 1. UWB antenna with dimensions in $\mathrm{mm}$ (front view) 


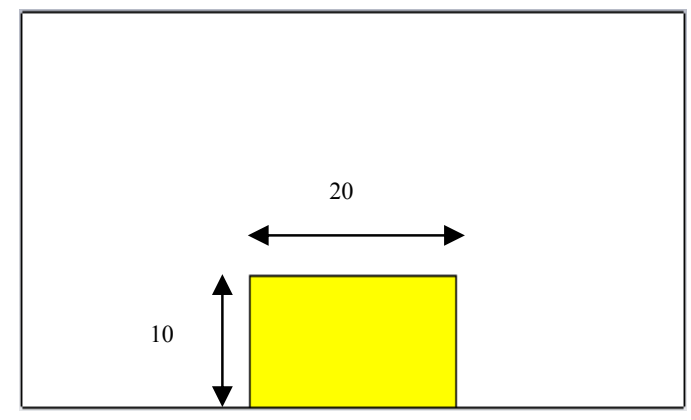

Figure 2. UWB antenna with dimension in $\mathrm{mm}$ (back view)

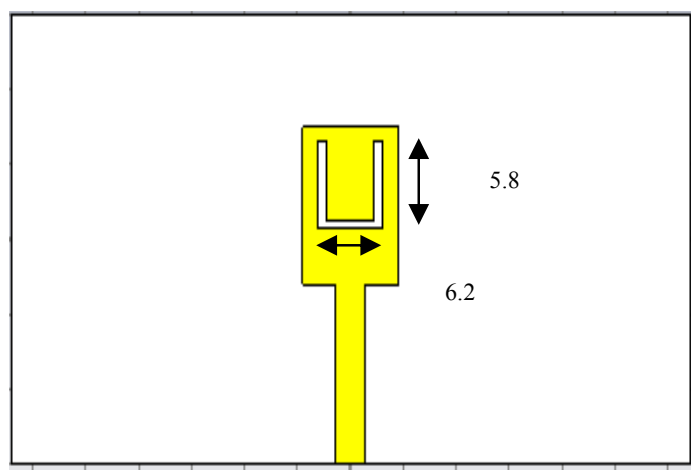

Figure 3. UWB antenna having slot with dimension in $\mathrm{mm}$

By increasing the distance between the elements of the antennas, the mutual coupling can be reduced. However, the distance between the antennas cannot be maintained too large, since MIMO systems have their major applications in Mobile terminals, laptops, MODEMs, WLAN Access Points etc., where miniaturization is the main task. Hence, we can achieve less mutual coupling by another means known as diversity concept. There are various diversity techniques like, spatial diversity, polarization diversity and pattern diversity for MIMO systems. In all these techniques, the mutual coupling can be mitigated more with the use of polarization diversity. The orthogonality of two distinct polarizations constructs independent and uncorrelated signals on each antenna. In the design of our system to achieve orthogonal polarization, one antenna is rotated to $90^{\circ}$ with respect to its adjacent element as shown in the Figure 7.The separation between the antennas is $12.8 \mathrm{~mm}$ which is $0.4 \lambda$. The antennas in the array have the same dimensions as mentioned in Section 2. The antennas are mounted on a substrate symmetrically with $\mathrm{gr}=4.3$, which in turn is mounted on a ground plane. The simulated results are shown in Figure 8 and 9 for $S_{11}$ and $S_{22}$ respectively, we can see that $S_{11}$ and $S_{22}$ curves show that both antennas are operating in the UWB region independently, also the insertion loss $\mathrm{S}_{12}$ between the two antennas is very low as shown in Figure 10.We also calculate the correlation coefficient and diversity gain for the two antenna array. Correlation coefficient formula using $\mathrm{S}$ parameters is given as in (2).

$\rho=\frac{\left|S_{11^{*} S 12+S 21^{*} S 22}\right|^{2}}{\left(1-\left|S_{11}\right| 2_{-}\left|S_{S 21}\right|{ }^{2}\right)\left(1-|S 22|^{2}-|S 12|^{2}\right)}$

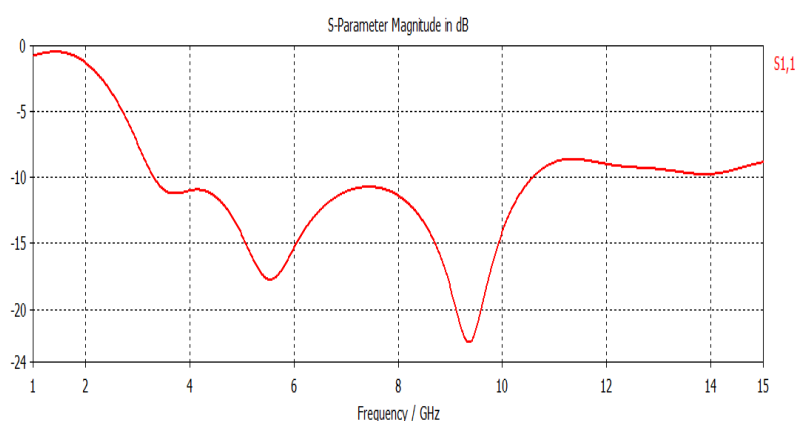

Figure 4. Simulated results for UWB antenna

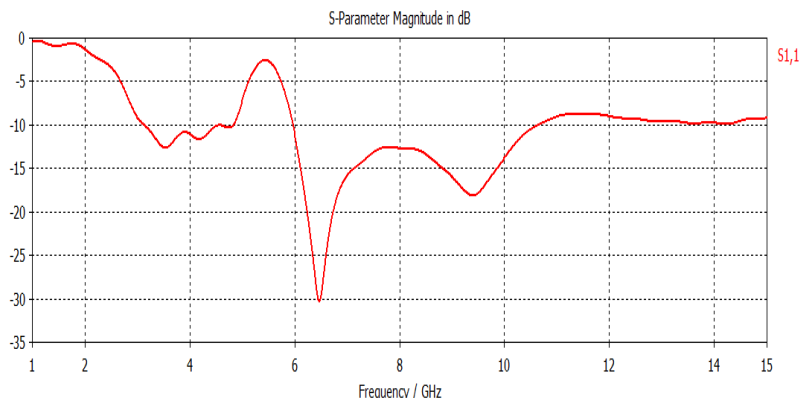

Figure 5. Simulated results for the notched UWB design

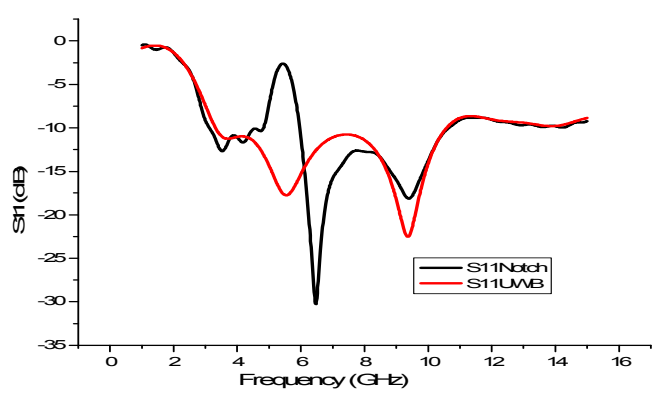

Figure 6. Comparative results for UWB with and without notch

Diversity gain is given by equation (3)

$$
D G=10 * \sqrt{1-(0.99 * \rho)^{2}}
$$

The correlation coefficient is calculated from the S-parameters and it comes out to be 0.00036 and diversity gain comes to be $9.95 \mathrm{~dB}$, which shows that antennas are operating independently of each other.

\section{B. Design of $2 X 2$ MIMO System}

We have designed the transmitting section, having two UWB antennas that are mutually independent of each other, now for the receiver side we take two notched antennas separated by the same distance as the transmitting antennas.

The idea behind taking UWB antennas at the transmitter and notched antennas at the receiver is that, from the $S_{11}$ plots in Figure 4 we observed that for UWB antenna lowest attenuation is at a frequency of $9.4 \mathrm{GHz}$ used for radar 
applications, these UWB antennas are used at the transmitter and at the receive sides notched antennas will remove the interference from WLAN band if any. The receiving section for the 2X2 System is shown in Figure 11 having notched antennas. Thus we have a four port system having two ports numbered 1 and 2 for transmitting side and ports 3 and 4 for the receiving side.

We can see the simulation results for the four port system in Figure 12. The channel matrix for the system can be calculated by observing $\mathrm{S}_{31}, \mathrm{~S}_{32}, \mathrm{~S}_{41} \mathrm{~S}_{42}$ from the plots, at a frequency of $9.4 \mathrm{GHz}$, these will be the coefficients of the channel matrix, as the $\mathrm{S}$ parameters simply denote the ratio of received and transmitted voltages, these ratios denote channel matrix coefficient.

The channel matrix for the $2 \mathrm{X} 2$ system can be written as in equation (4).

$$
H=\begin{array}{ll}
S 31 & S 32 \\
S 41 & S 42
\end{array}
$$

This channel matrix will be used for channel coding and OFDM transmission and reception in the next section.

\section{IV.UWB MIMO OFDM SYSTEM DESIGN}

\section{A. OFDM Basic}

OFDM is an efficient technique for transmitting data over frequency selective channels. The main idea behind OFDM is to divide a broadband frequency channel into a few narrowband sub-channels. Then, each sub-channel is a flat fading channel despite the frequency selective nature of the broadband channel. OFDM is a multicarrier modulation, To generate these parallel sub-carriers in OFDM, an inverse fast Fourier transform (IFFT) is applied to a block of $L$ data symbols. For a multipath environment, the transmitter sends pilot signals and the receiver uses them to estimate the channel. Then, the receiver uses the estimated path gains to coherently decode the data symbols during the same frame. So we add pilot signals for channel estimation.

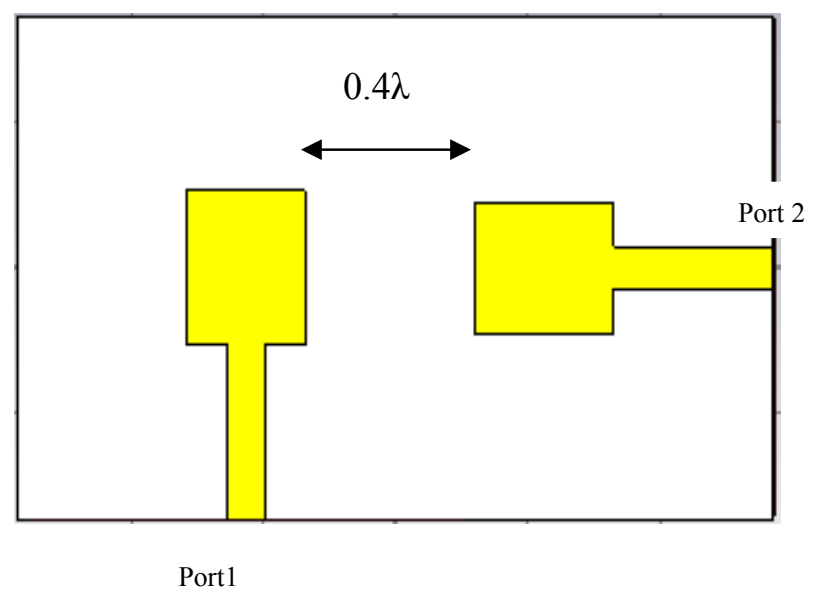

Figure 7. MIMO transmitting section having two antennas

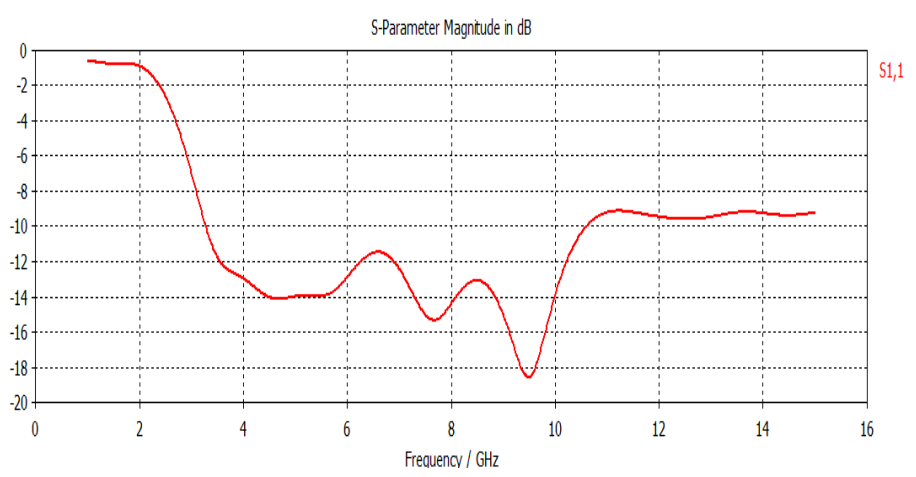

Figure 8. Plot for $\mathrm{S}_{11}$ showing UWB characteristic.

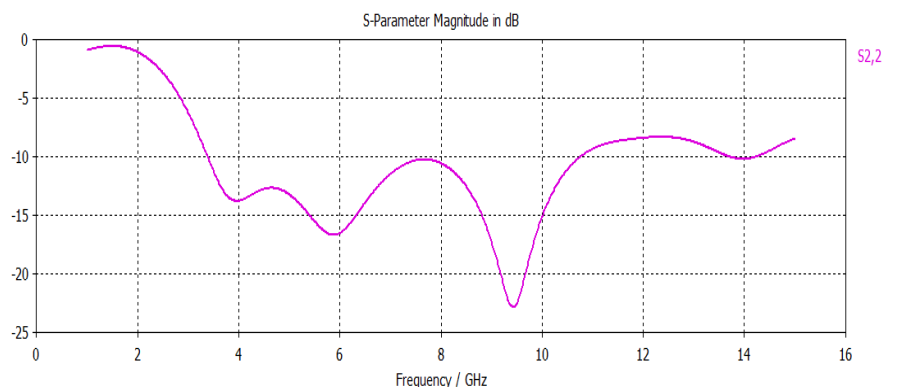

Figure 9. Plot for $\mathrm{S}_{22}$ showing UWB characteristic.

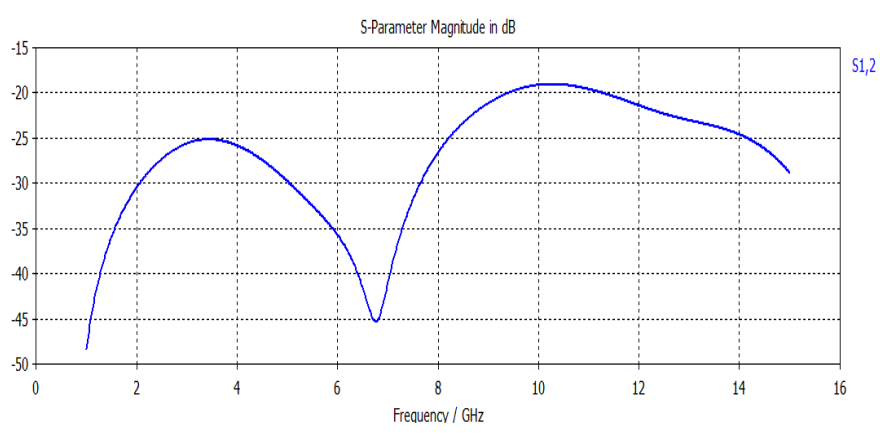

Figure 10. Plot for $\mathrm{S}_{12}$

To avoid ISI due to the channel delay spread, a few "cyclic prefix" (CP) symbols are inserted in the block. The effects of the cyclic prefix samples eliminate ISI and convert the convolution between the transmit symbols and the channel to a circular convolution. These cyclic prefix samples are removed at the receiver. After that pilot signals are removed Then, a fast Fourier transform (FFT) is utilized at the receiver to recover the block of $L$ received symbols.

\section{B. Generation of OFDM Symbol}

OFDM is a kind of frequency division multiplexing where modulation of OFDM symbols is done through orthogonal subcarriers. The UWB 3.1 to $10.6 \mathrm{GHz}$ band is divided into 14 bands, each with a bandwidth of $528 \mathrm{MHz}$ and devices are allowed to statically or dynamically select which bands to use for transmission. OFDM is a type of multi channel modulation that divides a given channel into many parallel sub-channels 
or sub-carriers, so that multiple symbols are sent in parallel so that each sub-carrier experiences a flat channel. In OFDM, $N$ orthogonal sub-carriers modulated by parallel data streams. Each baseband sub-carrier is of the form given by equation (5).

$$
\emptyset(t)=e^{j 2 \pi f t}
$$

where, $f$ is the frequency of sub-carrier and $\Phi$ forms an orthonormal basis function. One baseband OFDM symbol multiplexes $N$ modulated subcarriers as given by equation (6).

$$
s(t)=\frac{1}{\sqrt{N}} \sum_{k=0}^{N-1} x \emptyset(t)
$$

where, $\mathrm{x}$ is the complex data symbol taken from a BPSK constellation in our system, here $N$ is the number of subcarriers. The subcarrier frequencies are equally spaced which makes them orthogonal. For the implementation as in above equation it needs $N$ oscillators and DACs, which is of very high complexity. So, discrete time implementation of the equation is achieved using IDFT, which is further implemented by using IFFT and the frequencies are orthogonal because the basis function of Fourier transform is orthonormal. After that pilot signal insertion and cyclic prefix are inserted. The parallel symbols are converted to serial form. Then, utilizing the channel matrix of 2X2 MIMO system and random noise addition, the reverse process is carried out and finally the output bits are compared with the input bits to calculate bit error rate which is plotted against signal to noise ratios in $\mathrm{dB}$. The complete block diagram is shown in Figure 13. The coding for OFDM modulation has been done as follows; firstly, the random bits are generated, which is input to the system. These are converted to the points from a BPSK constellation after that serial to parallel conversion of data is done, the number of symbols are arranged in parallel.

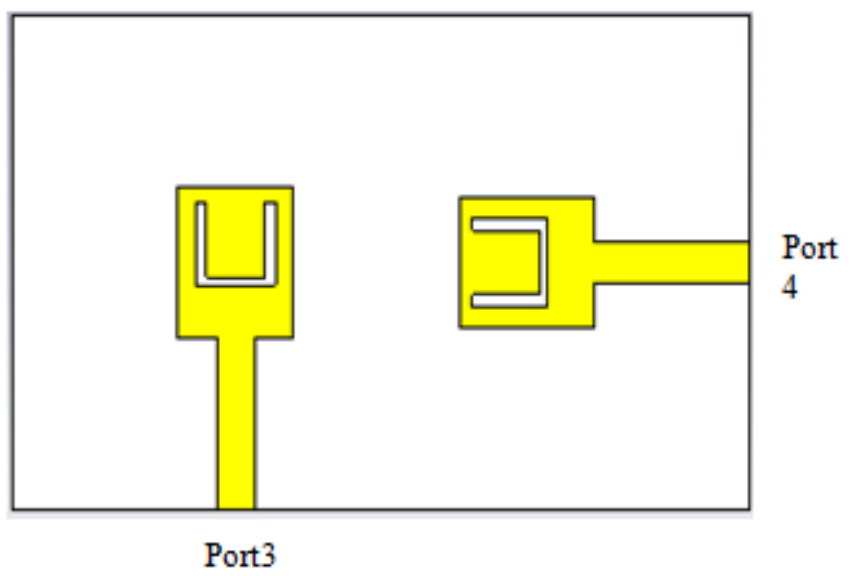

Figure 11. MIMO receiving section having notched antennas

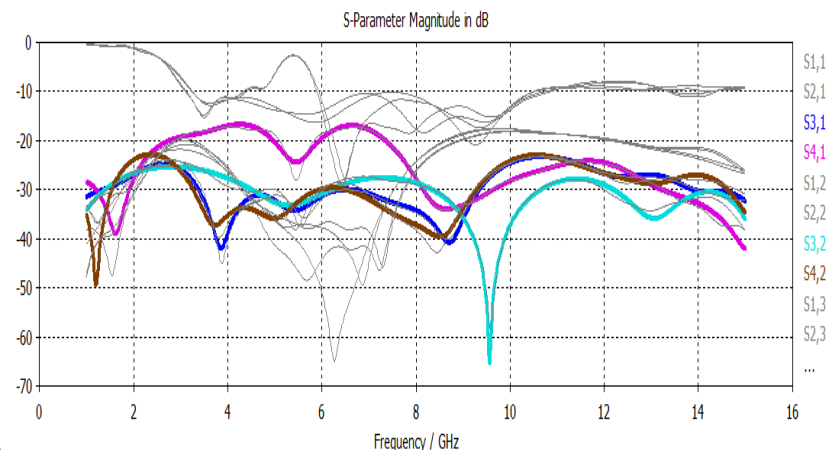

Figure 12. Overall Plots for the $2 \mathrm{X} 2$ system

Then inverse fast Fourier transform is done for each of the symbols. The number of subcarriers and number of bits in each symbol are taken to be equal. Then pilot insertion and cyclic prefix insertion is done for each of the symbols and again parallel to serial conversion is done, after that the serial bits are encoded to be transmitted in a $2 \times 2$ system. They are de-multiplexed into two separate streams and each stream is transmitted from an independent antenna. The channel matrix obtained from CST for a 2X2 system is used in coding the output from the $2 \mathrm{X} 2$ system with random noise added. The output is decoded serially from the two receivers. Now, the reverse process is carried out pilot removal and cyclic prefix removal is done, after this BPSK threshold detection is done. Finally the output bits are compared with input bits for bit error rate which is calculated at different signal to noise ratios. The curve is as shown in Figure 14 for a distance (d) of $16 \mathrm{~mm}$ between the transmitter and receiver sections which is greater than the far field distance. We can see that BER decreases with increase in signal to noise ratio.

The, BER plots has also been calculated for different distances between transmitter and receiver sections.
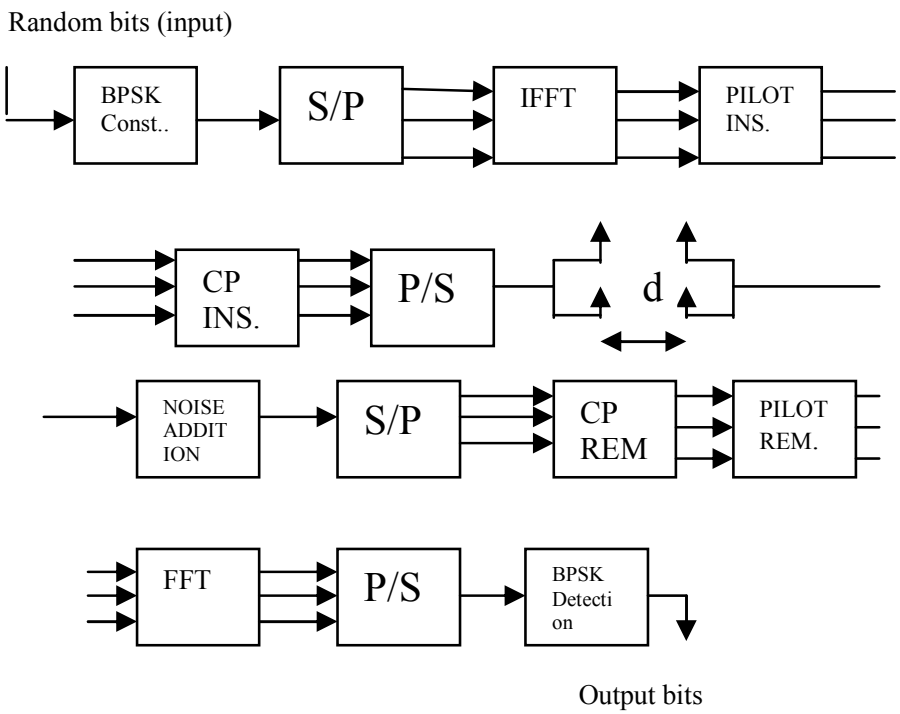

Figure 13. Block Diagram for MIMO-OFDM process 
In the plot of Figure 15, data1, data2, data3 data4 data5, corresponds to a different BER plots for distance of $16 \mathrm{~mm}$, $50 \mathrm{~mm}, 100 \mathrm{~mm}, 150 \mathrm{~mm}, 300 \mathrm{~mm}$ respectively. We can see that as the distance between transmitter and receiver increases BER increases.

\section{CONCLUSIONS}

A new methodology has been defined to estimate the performance analysis of a 2X2 MIMO OFDM system operating on UWB using practical antennas designed on CST Microwave studio, the obtained channel matrix from CST has been used for further coding MIMO OFDM system instead of using a random channel matrix.

The system has been designed for $9.4 \mathrm{GHz}$ frequency that is used for RADAR applications along with notching of WLAN band at the receiver side, to eliminate interference if any. The BER vs SNR curves are shown for the system, as expected the bit error rate decrease by increasing signal to noise ratio and also it can be seen that bit error rate is increased by increasing the distance between transmitter and receiver sections. Thus, a practical system is designed and results are coming as expected.

\section{REFERENCES}

[1] G.J.Foschini, "Layered space-time architecture for wireless communication in a fading environment when using multi element antennas," BLTJ, Autumn, 1996

[2] FCC. Revision of part 15 of the commission's rules regarding ultra wideband transmission systems. Technical report, Federal Communications Commission's, ET Docket, 2002.

[3] Ruan Cheng-li. "UWB antenna theory and technology[M]".Harbin industrial university press, 2005

[4] T. Svantesson. "On the Capacity and Correlation of Multi-Antenna Systems Employing Multiple Polarizations". Proceedings of IEEE Antennas and Propagation Symposium vol. 3, pp. 202-205, June 2002

[5] Emami-Forooshani 1 S. Noghanian "Semi-deterministic channel model for MIMO systems Part-II: results “ IET microwaves, antennas \& propagation vol4 pp 26-34 2010

[6] Matilde Sanchez-Fernandez, Eva Rajo-Iglesias, Oscar Quevedo-Teruel M. Luz Pablo-Gonzalez. "Spectral Efficiency in MIMO Systems Using Space and Pattern Diversities Under Compactness Constraints" IEEE T1637-1645, May 2008.

[7] Mukherjee and Hyuck M. Kwon. "Compact Multi-user Wideband MIMO System Using Multiple-Mode Microstrip Antennas" Proceedings of Vehicular Technology Conference Spring 2007, pp584588, Apr 2007

[8] Waldschmidt, C. Kuhnert, S. Schulteis, and W. Wiesbeck, "Compact MIMO-Arrays Based on Polarization-Diversity". Proceedings of IEEE Antennas and Propagation. Symp., vol2 , pp. 499-502, June 2003.

[9] M. A. Jensen, J. W. Wallace, "A review of antennas and propagation for MIMO wireless communications", IEEE Trans. Antennas Propagation., vol. 52, pp. 2810-2824, Nov. 2004

[10] T. Svantesson. "On the Capacity and Correlation of Multi-Antenn Systems Employing Multiple Polarizations". Proceedings of IEEE Antennas and Propagation Symposium vol. 3, pp. 202-205, June 2002

[11] Abouda, A. A. and S. G. H"aggman, "Effect of mutual coupling capacity of MIMO wireless channels in high SNR scenario," Progress In Electromagnetics Research, PIER 65, 27-40,

[12] R. Mallahzadeh, S. Es'haghi, and A. Alipour, "Design of an E shaped MIMO antenna using IWO algorithm for wireless applications at 5.8GHz." Progress In Electromagnetics Research, PIER 90, 187-203, 2009

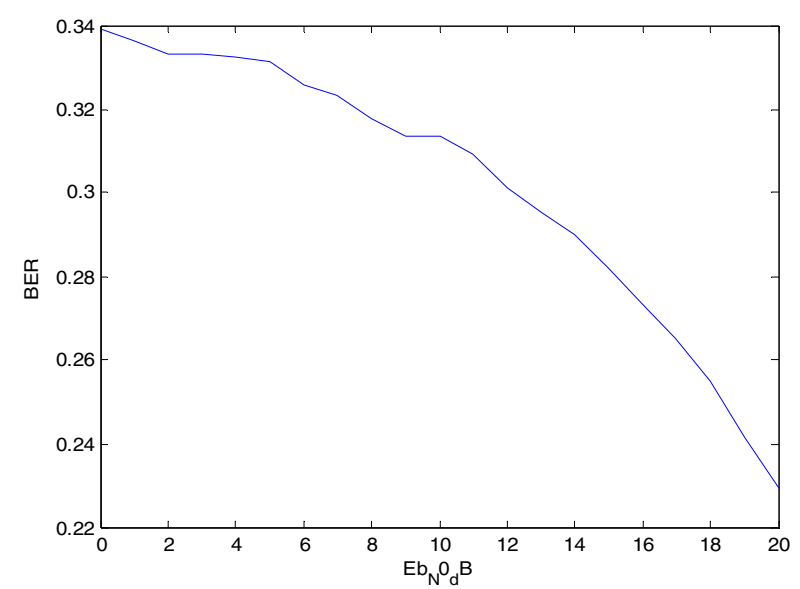

Figure 14. BER vs $\mathrm{SNR}(\mathrm{dB})$ for a distance of $16 \mathrm{~mm}$ between transmitter and receiver section

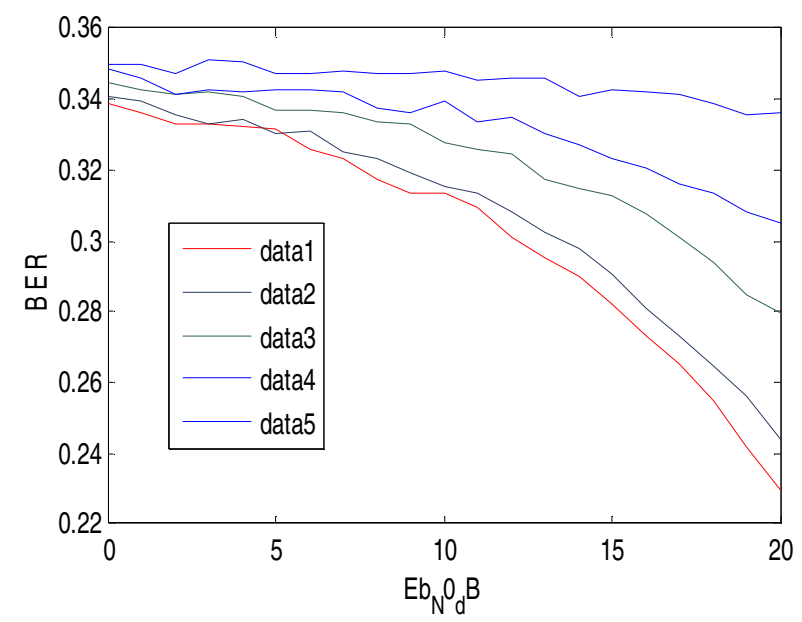

Figure 15. BER vs SNR(dB) for different distance between transmitter and receiver sections

[13] L. Yang and G. B. Giannakis, "Ultra-wideband communications: An idea whose time has come," IEEE Signal Process. Mag., vol. 21, no. 6 , pp. 26-54, Nov. 2004.

[14] L. Yang, "Low-complexity diversity receiver for single/multi-band UWB," in Proc. IEEE Int. Workshop Signal Process. Adv. Wireless Commun.,New York, Jun. 5-8, 2005, pp. 1053-1057.

[15] C. Chang, J. C. Liu, and M. Y. Liu, "A novel tulip-shaped monopoleantenna for UWB applications", Microw. Opt. Technol. Lett., vol. 48,pp. 307--312, 2006.

[16] Eva Antonino-Daviu, Marta Cabedo-Fabrés, Miguel FerrandoBataller,and Vicent Miquel Rodrigo Peñarrocha,'Modal,Analysis and Design of Band-Notched UWB Planar Monopole Antennas", IEEE Transactions on Antennas and Propagation, Vol. 58, No. 5, May 2010.

[17] G. Aiello, J. Foerster, A. Batra, J. Balakrishnan, A. Dabak. "Design of a multiband ofdm system for realistic uwb channel environments". IEEE Trans. Microwave Theory Tech., 2004.

[18] Batra, J. Balakrishnan, G. R. Aiello, J. R. Foerster, and A. Dabak, "Design of a multiband OFDM system for realistic UWB channel environments, 'IEEE Trans. Microw. Theory Tech., vol. 52, no. 9, pp. 2123-2138, Sep. 2004. 


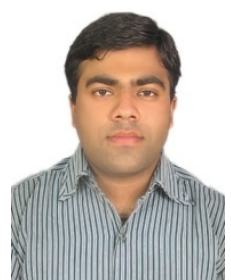

Harshal Nigam (B.E 2011) received the B.E degree in Electronics and Communication Engineering from College of Technology and Engineering, Maharana Pratap University of Agriculture and Technology, Udaipur, Rajasthan, India in 2011. Currently, he is pursuing his Masters degree in Digital Communication from University College of Engineering, Rajasthan Technical University, Kota, Rajasthan India. His research interest focuses on RF Communication, UWB antenna system design and also in the field of Wireless communications.

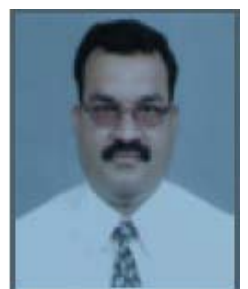

Mithilesh Kumar (B.E. 1992-M.Tech. 1999-Ph.D 2011) received the B.E degree in Electronics and Communication Engineering from Government Engineering College, University of Rajasthan, Kota, Rajasthan, India in 1992, M.Tech. degree in Communication and Radar Engineering in 1999 and $\mathrm{Ph} . \mathrm{D}$. degree in RF Communication Engineering from the Indian Institute of Technology Delhi, India in 2011. He served as a Lecturer (1993-2000), Sr. Assistant Professor (2000-2008) and Associate Professor (2008-2013) at Rajasthan Technical University, Kota, Rajasthan, India. Currently he is a Professor (2013-till date) at the same university. He is author/ co-author of 85 Research papers in referred Journals, International and National conferences. His current research interests include RF Communication, UWB Active Antenna, Microstrip Antenna and Microwave Device Designing Technology. Dr. Kumar received "First prize best student paper platinum award" in 2009 at the IEEE International Conference on Antennas, Propagation and Systems (INAS-2009) held at Johor Bahru, Malyasia from December 3-5, 2009. 